\title{
The Conductivity of Indium Phosphide Irradiated by Fast Electrons
}

\author{
Sh. Sh. Rashidova \\ Institute of Physics, Azerbaijan National Academy of Sciences, Baku, Azerbaijan \\ Email: sh.sh.rashidova@gmail.com
}

Received May 27, 2013; revised June 29, 2013; accepted July 24, 2013

Copyright (C) 2013 Sh. Sh. Rashidova. This is an open access article distributed under the Creative Commons Attribution License, which permits unrestricted use, distribution, and reproduction in any medium, provided the original work is properly cited.

\begin{abstract}
In this work, studied electrical conductivity(s) and annealing of radiation defects in crystals of $n$-InP are irradiated by electrons energy of $6 \mathrm{MeV}$ and doses of $10^{17} \mathrm{el} / \mathrm{cm}^{2}$ (centimeter) and $2 \times 10^{17} \mathrm{el} / \mathrm{cm}^{2}$ (centimeter). It is shown that alongside point defects (in the form of complexes with impurity atoms in crystals of $n$-InP) also form the complex defects of the type of disordered areas, annealing of which proceeds at $T>300^{\circ} \mathrm{C}$ that binds accumulating radiation defects.
\end{abstract}

Keywords: Antistructural Defects; Activation Energy; Indium Phosphide; Radiothermoluminescence Method

\section{Introduction}

Investigations of the effects of fast electrons irradiation on the electrical properties of InP are of scientific and practical interest. From the scientific point of view, such investigations give information about the origin of radiation-induced defects in InP. From the practical viewpoint, it should note the technology of fabrication of transistors, solar elements and solar cells on InP.

The introduction of high-energy ions in the crystal impurities is accompanied by disordering of the crystal lattice. For elimination of radiation defects and electrical activation impurities, annealing crystals were applied.

\section{Experimental Procedure}

Results of researches of electric conductivity of irradiated by electrons $6 \mathrm{MeV}$ with concentrations of $n=10^{18}$ $\mathrm{cm}^{-3}$ crystals of $n$-InP. Irradiation was performed on a linear accelerator type EBA-6 MeV (EBA-Electron beam acceleration). Accelerator worked at a frequency of 50 $\mathrm{Hz}$ in the impulsive mode.

Pulse duration equal to 2.5 ISS. The samples had thickness 1000 - $2000 \mathrm{mkm}$ (micrometer). Samples of single crystals of $n$-InP were obtained by Czochralski method. Irradiation was performed at room temperature. Carry out measurement of electric conductivity(s) before and after irradiation in the temperature range from $80 \mathrm{~K}$ to $300 \mathrm{~K}$.

Afterwards, the samples underwent isochronal anneal- ing (heating time $10 \mathrm{~min}$.) in the temperature range from $50^{\circ} \mathrm{C}$ to $480^{\circ} \mathrm{C}$.

\section{Results and Their Discussion}

In Figure 1, it is shown that the temperature dependence of the conductivity in InP crystals with concentration $n=$ $10^{18} \mathrm{~cm}^{-3}$ before (curve 1) and after electrons irradiation $E=6 \mathrm{MeV}$, doses $D=10^{17} \mathrm{el} / \mathrm{cm}^{2}$ (curve 2) and $D=2 \times$ $10^{17} \mathrm{el} / \mathrm{cm}^{2}$ (curve 3 ).

As can be seen from the temperature dependence of the electrical conductivity of crystals in $n$-InP irradiated by fast electrons with a high dose of conductivity reaches a minimum of about $T=170 \mathrm{~K}$. Decrease of the electrical conductivity in the temperature interval from 100 to $170 \mathrm{~K}$ cannot determine mobility. This behavior conductivity might be due to the deep-lying levels of radiation entered defects. At higher temperatures (above $170 \mathrm{~K}$ ) capture of electrons is reduced. The activation energy is estimated by the high temperature of part $E=0.12 \mathrm{eV}$ which is in good agreement with literature data [1].

The electron irradiation by high-energy enters into crystals InP a high number of donors and acceptors of the high degree of their concentration also influence of irradiation formed stable of interstitial phosphorus [2].

As can be seen from figure irradiation dose $10^{17} \mathrm{el} / \mathrm{cm}^{2}$ (centimeter) conductivity decreases the slope of the curve disappears completely. But on the second stage of irradiation, the dose $D=2 \times 10^{17} \mathrm{el} / \mathrm{cm}^{2}$ (centimeter) reduce conductivity increases, observed the minimum (curve 3 ) 
at a temperature of $170 \mathrm{~K}-180 \mathrm{~K}$. This is probably due to the deep-lying levels of radiation defects caused by irradiation [3] which compensates for the electrical conductivity of the crystal. At higher temperatures about 180 $\mathrm{K}$ electronic traps are exempt. Activation energy is estimated from the high temperature part (Figure 1 curve 3) $E=0.14 \mathrm{eV}$, which agrees well with the temperature data [4].

This is confirmed in work [5] a model where the electron irradiation produces $\mathrm{M}$ centers in $n$-InP. These centers are located in two charging status with the activation energy $E=0.09 \mathrm{eV}$ and $E=0.14 \mathrm{eV}$. These levels have been detected earlier us [6,7] by method RTL in these crystals irradiated with gamma quanta at a temperature of $170 \mathrm{~K}, 182 \mathrm{~K}$ with the activation energies $E_{a}=0.09 \mathrm{eV}$ and $0.14 \mathrm{eV}$ respectively. Levels with $E_{a}=0.09 \mathrm{eV}$ lie within $0.1 \mathrm{eV}$ from the edges of the conduction and valence band are associated with the formation of the complex migration of these vacancy in the material with the admixture in the form of a vacancy is impurity. Level with the energy of activation $0.14 \mathrm{eV}$ appeared in the crystal $\mathrm{InP}<\mathrm{Sn}>$ and for increasing doses up to $3.5 \mathrm{kGr}$ this peak is shifted to $0.01 \mathrm{eV}$ towards higher energies.

This phenomenon can be associated with the formation of complexes entered defects and interstitial phosphorus atoms $\mathrm{I}_{\mathrm{P}}$.

At annihilation of radiation defect also may decrease the nature of the lattice, however, when the concentration of antistructural defects donors type $\mathrm{P}_{\text {In }}$ (atoms $\mathrm{R}$ nodes sub lattice In) greater concentration of charge carriers in the InP. It can be noted that non-structure defects formed during the irradiation of particles single crystals $\mathrm{A}^{3} \mathrm{~B}^{5}$ have a more complex structure than those formed during the crystal growth.

These defects can significantly affect the electrical properties of these materials. Figure 2 shows the temperature dependence of the electrical conductivity $(\sigma)$ for sample InP before and after $6 \mathrm{MeV}$ irradiation with $D=2$ $\times 10^{17} \mathrm{el} / \mathrm{cm}^{2}$ and after isochronal annealing in the temperature range from $50^{\circ} \mathrm{C}$ to $480^{\circ} \mathrm{C}$.

As is seen from figure 2 after irradiation, the sharp decrease of the electrical conductivity in the beginning (170 $\mathrm{K})$, and then increase with the increase of temperature independence $\sigma(T)$ (curves 3,4).

The first slope $(E=0.33 \mathrm{eV})$ corresponds to a deeper level, the second one $(E=0.41)$ is probably connected with the of conduction due the formation of disordered regions or to conpensation (after irradiation).

The disordered regions in irradiated semiconductors which modulates the energy bands and strongly effects in the temperature dependence of electrical conductivity. It can be assumed that after the irradiation the disordered regions formed in the sample overlap, and this reduce the efficiency of disordered regions are released as the an-

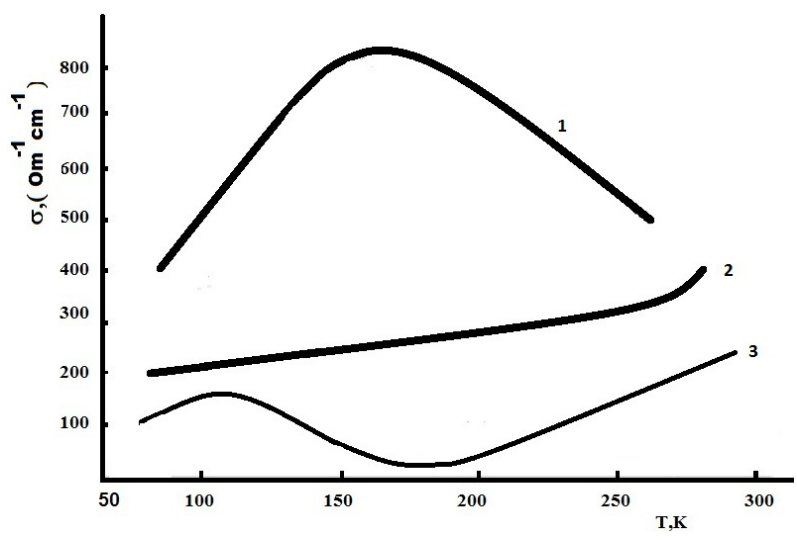

Figure 1. Temperature dependence of the conductivity for crystals $n$-InP before irradiation; (curve 1) after irradiation with $6 \mathrm{MeV}$ electrons with doses $10^{17} \mathrm{el} / \mathrm{cm}^{2}$ (curve 2) and 2 $\times 10^{17} \mathrm{el} / \mathrm{cm}^{2}$ (curve 3).

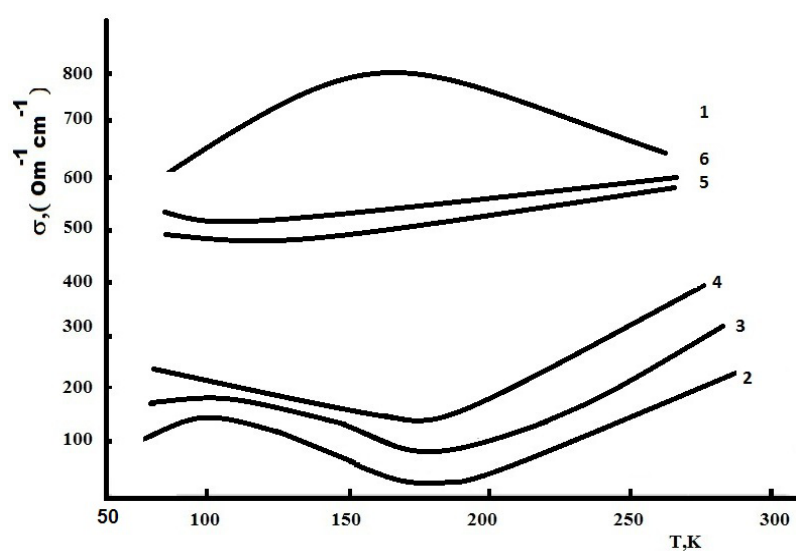

Figure 2. Temperature dependency of the electrical conductivity for sample InP: (1) before irradiation; (2) after irradiation with electron energy $E=6 \mathrm{MeV}$ with dose $D=2 \times$ $10^{17} \mathrm{el} / \mathrm{cm}^{2}$ (centimeter); after $10 \mathrm{~min}$ isochronal annealing at $\mathrm{T}=330^{\circ} \mathrm{C} \mathrm{(3),} 380^{\circ} \mathrm{C} \mathrm{(4),} 430^{\circ} \mathrm{C} \mathrm{(5),} 480^{\circ} \mathrm{C} \mathrm{(6)}$.

nealing temperature increases.

The isochronal annealing of the irradiated sample gradually recovers the electrical conductivity.

Annealing was performed in quartz ampoules. As is seen from Figure 2 radiation enters the crystal InP a large number of donors and acceptors, also the influence of irradiation is formed stable interstitial atom of phosphorus, the high degree of their compensation. Irradiation is formed by point defects and is stable at room temperature. With the increase of the annealing temperature degree of compensation is reduced.

Deep levels of the created pairs vacancy-antistructural defects $\left(\mathrm{V}_{\mathrm{In}} \mathrm{In}_{\mathrm{P}}\right)$ the nature belong to defects in crystals with the structure of zinc blende. These deeper levels are within the width of the forbidden zone.

In the presence of crystals of dopants or radiation defects own point defects can form with them complexes.

When ionization defect is a leap nearest phosphorus 
atom to place vacancies India and complex turns into a $\mathrm{P}_{\mathrm{In}}^{+} \mathrm{V}_{\mathrm{P}}^{+} \mathrm{P}_{\mathrm{In}}^{++} . \mathrm{P}_{\mathrm{In}}$ is antistructural defects of donors' type.

\section{Conclusions}

By measuring electrical conductivity, we have found that after irradiation of $n$-InP crystals with $6 \mathrm{MeV}$ electrons, there arise disordered regions with a space charge, radiation-induced charged defects.

It is also assumed that after electron irradiation, owing to compensation, there appears hopping conduction in InP at $T<170 \mathrm{~K}$, in $n$-InP to the recharging of the radiation defects available and to the deformation of secondary defects.

Apart from the processes described above, during annealing, secondary defects are formed which would lead to the reverse annealing of conductivity.

\section{REFERENCES}

[1] P. K. Chakraborty and K. P. Ghatak, Journal of Applied
Physics, Vol. 89, 2001, pp. 1075-1081. http://dx.doi.org/10.1063/1.1330253

[2] N. Q. Kolin, D. I. Merkurisov and S. P. Solovyev, FTS, Vol. 34, 2000, in press.

[3] N. P. Kekelidze, Radiation Effects, 1977, Vol. 32, pp. 113-119. http://dx.doi.org/10.1080/00337577708237465

[4] J. L. Benton, M. Levinson, A. T. Macrander, H. Tenikin and L. C. Kimerling, Applied Physics Letters, Vol. 45, 1984, p. 566. http://dx.doi.org/10.1063/1.95282

[5] J. Suski and J. C. Boiurgoins, Journal Applied of Physics, Vol. 54, 1983, p. 2852. http://dx.doi.org/10.1063/1.332277

[6] Y. W. Zhao, Z. Y. Dong, M. L. Duan and W. R. Sun, European Physical Journal Applied Physics, Vol. 27, 2004, pp. 167-169. http://dx.doi.org/10.1051/epjap:2004096

[7] Sh. Sh. Rashidova, Journal of Engineering Physics and Thermophysics, Vol. 84, 2011, pp. 479-482. http://dx.doi.org/10.1007/s10891-011-0494-8 